Nans
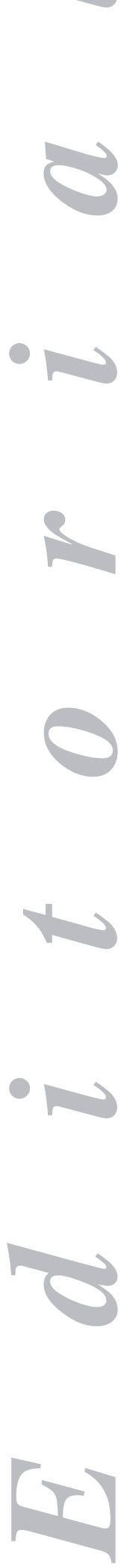

\title{
Personalized medicine: inevitable
}

Gene testing to identify patients for whom certain drugs will be effective or to ensure drug safety belong to the diagnostic tools of modern medicine. Although the pharmaceutical industry previously would wish their medicines to be used by as many patients as possible, it now recognizes the value of tailoring medicine on specific genetic or biologic markers to specific individual patients. Molecular diagnostics have stimulated pharmaceutical companies to create molecular diagnostic units. By the selection of patients who will benefit from tailor made medicines, the way how we treat disease will alter. When we decided to study medicine, we wanted to give the best care to our patients and dreamed of knowing each patient and each family well enough to advise the most indicated medicine or simply advise to wait and see. The advancement of "super specialization" and medical technology did not allow this dream. Also the medical specialist was too busy and this care has been taken over by family practitioners.

The advances in genetics in the year 2000 created a new impetus and vision to what is now called personalized medicine. Personalized medicine makes it possible to prescribe the right drug for the right patient at the right time. In due course personalized medicine will enable to save a considerable amount of money and time since the trial and error part of medical treatment can be avoided. This will help to improve the management, prevent side effects and make health care more efficient and cost effective in the future. The new area of personalized medicine does not only apply to genetics but also to the technology of communication, the internet and the protection of privacy of data of patients in computer systems.

In the field of inflammatory bowel disease, the capability to genotype hundreds of thousands of common sequence variants across the genome permitted the discovery of specific alleles involved in susceptibility. For this however, large cohorts of patients and controls were needed. A good example is the genome-wide association study of 14,000 cases of seven common diseases and 3,000 shared controls undertaken in the British population using the Affymetrix GeneChip 500K Mapping Array. In this study, the authors found in approximately 2,000 individuals with Crohn's disease a set of 9 genes significantly associated with the disease (1). Another large study of combined data from three studies on Crohn's disease (2) found significant evidence for 21 additional loci and in addition it confirmed 11 previously reported loci (2). This new information has contributed to change the view on the pathogenesis of Crohn's disease but it has not yet reached the clinical field. In the latter studies the SNPS selected did not represent the gene involved and the function of many of these genes is still unknown. However, in these multifactorial chronic intestinal diseases, thousands of individuals would be needed to discover the effect of genes involved in predisposition of disease and in the response to treatment. 
This year marks the 10th anniversary of the announcement by Dr. Collins and Dr. Venter on the completion of the draft sequence of the human genome, although it took another three years to complete the project. Up to date, few medicines have been included in the armamentarium of the so called targeted therapies. Some of these are in cardiovascular medicine, breast cancer and colon cancer. Although it is known that more than 200 product labels recommend genetic testing before prescription or point to the influence of genetic variation on its drug response or drug safety.

Genetic medicine finds itself in a crisis in the year 2010. One of the enlightened forces to promote personalized medicine, Dr. Francis Collins, at present the Director of the NIH in Bethesda, USA, has been criticized on the issue of the high cost of genetic medicine and the delay in delivering translational results for clinical medicine. The signing by President Obama of the landmark health care reform legislation some months ago represented a first major victory for personalized medicine. This is not a general shared opinion. Many medical scientists feel that fewer funds should be allocated to genetics and more funds to epidemiological research.

With this background I like to thank the editorial board for publishing in this issue of the Spanish Journal of Digestive Diseases a preliminary report on gene variables and response to a biological drug in patients with Crohn's disease by Manuel Barreiro et al., although the observations apparently are insignificant since they were made in only 24 patients and only three genes were studied. I mention "apparently" because the contribution in this area reminds me of the first publications of our group in $1995(3,4)$ when trying to understand genetic predisposition in IBD and multiple sclerosis. Progress of clinical science is a slow process. In 1995 the therapy with monoclonal antibodies had not yet been introduced. Since then biological therapies have shown great efficacy in the treatment of Crohn's disease, particularly in severe cases and in patients who fail to respond to conventional therapies such as corticosteroids. Adalimumab ${ }^{\circledast}$ is relatively new in the field of IBD. The authors observed in 24 patients with Crohn's disease from Galicia, Spain that $75 \%$ of the patients responded to Adalimumab ${ }^{\circledR}, 16.7 \%$ lost response to treatment and $12.5 \%$ terminated treatment due to adverse events. A search for genes to explain these results is obvious. The authors hypothesized that mutations in Crohn's disease susceptibility genes, involved in the regulation of the immune response, may influence treatment (5). None of the three genes chosen in this study were found to be predictive for differential clinical response to treatment with ${ }^{\circledR}$. In the discussion of the article the authors mentioned some of the drawbacks of the study and concluded that subsequent studies should be performed in a larger population. The main message for clinicians is the importance of collecting DNA from patients to contribute to the development of personalized medicine. It is important to obtain written consent of the patients, but as important is to explain to them the significance of genetic studies in tailored medicine. Full sequencing of DNA of each patient will soon become available and affordable. When available, every patient will be important for investigation. Properly recorded clinical findings and follow up will help the geneticists to predict response and to avoid side-effects.

New challenges will have to be addressed. Not only the cost of a full sequencing of the human genome will resolve the issue, the clinical translation of genetic risk estimates must be determined which will require expertise. Integrated analysis of a complete human genome in a clinical context of a patient with a family history of vascular disease and early sudden death has been performed for the first time. Rare variants were found in three genes that are clinically associated with sudden cardiac death. This means that physicians can screen patients at risk for heart attack in order to determine the most likely effective approach (6). Another example; Due to a genetic variation, 
some patients do not metabolize Clopidogrel normally and will not respond to treatment, they are Clopidogrel resistant. It has been suggested that whole genome sequencing can yield useful and clinically relevant information for such patients (7). For the specialist is gastroenterology a more relevant illustration refers to metastatic colorectal carcinoma. The Food and Drug Administration (FDA) in the U.S. has approved labeling changes for both Panitumumab and Cetuximab to indicate that these drugs should not be used by patients with mutations to the gene located on chromosome 12p12.1, known as KRAS. This gene encodes a protein that is a member of the small GTPase superfamily. A single amino acid substitution is responsible for an activating mutation which occurs in about $35 \%$ of patients. Avoiding use of Cetuximab as a first-line treatment for patients with this mutation could save patients' suffering and avoid considerable negative financial consequences. Patients with metastatic colorectal carcinoma carrying activating mutations of the KRAS gene have proven not to benefit from treatment with anti-EGF receptor monoclonal antibodies. Mutation testing of metastatic colorectal carcinoma patients is mandatory in the clinical setting to select the appropriate therapy (8).

A new era of genomics based on identification of rare variants that confer disease risk in individual patients is approaching. As pointed out by Lifton (9), the complete sequence of the human genome and the spectacular reductions in the cost of DNA sequencing, individual genomes are on the horizon. In an in-depth editorial, Ormond et al., has drawn attention to several new aspects. Even if a patient's whole-genome sequence is properly interpreted, the resulting information needs to be successfully conveyed to the patient. How will we do that? (10). Ormond et al. are optimistic about the value of whole genome sequencing in medical practice and have summarized six practical considerations demonstrating that implementation of such testing will be challenging. In part because as they correctly indicate, it is often assumed that information is good and more information is better, although more information can sometimes be counterproductive.

In the U.S., Hamburg and Collins have recently summarized the agenda of the path for personalized medicine. They have pointed out that real progress will come when clinically beneficial new products and approaches are incorporated into clinical practice. As the field advances, they expect to see more efficient clinical trials based on a more thorough understanding of the genetic basis of disease. They anticipate that some previously failed medications will be recognized as safe and effective and will be approved for subgroups of patients with specific genetic markers(11).

Similar to the introduction of "the internet" that by now has reached most of the poor countries in the world, I believe that full sequencing of individual genomes will be widely available in the next decade. The knowledge of the interaction of the human genome with the intestinal microbiota and other environmental factors will help the clinician to prescribe the right drug for the right patient at the right time. This is crucial for both the patient and the national health systems of all countries. My view at this moment is that personalized medicine is here to stay no matter how imperfect it still is in the year 2010. Personalized medicine is inevitable. It is our responsibility to get educated in genomic medicine in order to help and to educate our patients. The old Chinese proverb "a journey of a thousand miles must begin with a single step" has proven its truth.

A. S. Peña

Emeritus professor "VU" University Medical Centre. Amsterdam, The Netherlands 


\section{References}

1. Genome-wide association study of 14,000 cases of seven common diseases and 3,000 shared controls. Nature 2007; 447(7145): 661-78.

2. Barrett JC, Hansoul S, Nicolae DL, Cho JH, Duerr RH, Rioux JD, et al. Genome-wide association defines more than 30 distinct susceptibility loci for Crohn's disease. Nat Genet 2008; 40(8): 955-62.

3. Bioque G, Crusius JB, Koutroubakis I, et al. Allelic polymorphism in IL-1 beta and IL-1 receptor antagonist (IL-1Ra) genes in inflammatory bowel disease. Clin Exp Immunol 1995; 102(2): 379-83.

4. Crusius JB, Pena AS, Van Oosten BW, Bioque G, Garcia A, Dijkstra CD, et al. Interleukin-1 receptor antagonist gene polymorphism and multiple sclerosis. Lancet 1995; 346(8980): 979.

5. Barreiro-de Acosta M, Ouburg S, Morré SA, Crusius JBA, Lorenzo A, Potel J, et al. NOD2, CD14 and TLR4 mutations do not influence response to adalimumab in patients with Crohn's disease: A preliminary Report. Rev Esp Enferm Dig 2010; 102(10): 591-4.

6. Ashley EA, Butte AJ, Wheeler MT, Chen R, Klein TE, Dewey FE, et al. Clinical assessment incorporating a personal genome. Lancet 2010; 375(9725): 1525-35.

7. Cuisset T, Cayla G, Silvain J. Clopidogrel resistance: what's new? Arch Cardiovasc Dis 2010; 103(6-7): 34953.

8. Carotenuto P, Roma C, Rachiglio AM, Tatangelo F, Pinto C, Ciardiello F, et al. Detection of KRAS mutations in colorectal carcinoma patients with an integrated PCR/sequencing and real-time PCR approach. Pharmacogenomics. 2010;11(8):1169-79.

9. Lifton RP. Individual genomes on the horizon. N Engl J Med 2010; 362(13): 1235-6.

10. Ormond KE, Wheeler MT, Hudgins L, Klein TE, Butte AJ, Altman RB, et al. Challenges in the clinical application of whole-genome sequencing. Lancet 2010; 375(9727): 1749-51.

11. Hamburg MA, Collins FS. The path to personalized medicine. N Engl J Med 2010; 363(4): 301-4. 